nature

medicine

\title{
Of men, not mice
}

\section{A recent study showing that mice do not reproduce the patterns of gene expression induced by human inflammatory disease has provoked renewed discussion of the validity of animal models in translational research.}

A nimal models have long been important for dissecting the underlying mechanisms of many human diseases, as well as in transitioning promising candidate therapies from bench to bedside. However, there is a growing awareness of the limitations of some widely used disease models, a concern reinforced by a recent systematic study comparing transcriptional responses to inflammatory insults in mice and humans (Proc. Natl. Acad. Sci. USA 110, 3507-3512, 2013).

The Inflammation and Host Response to Injury, Large Scale Collaborative Research Program compared blood leukocyte gene expression profiles that were elicited by various inflammatory challenges, including sepsis, trauma, burns and endotoxemia, using both previously published and newly generated data sets from mouse models and human subjects. Although the transcriptional responses across the diverse conditions were similar among the human data sets, suggesting a common pathway in humans independent of the initiating stress, the gene expression changes in the corresponding mouse models significantly differed both from each other and from the human conditions. Highlighting just how poorly the mice reflected the human disease, the correlations of the gene changes in the mouse models with their human disease counterparts came close to those expected by random chance alone.

This study has its caveats. Importantly, as only a single strain of mice was examined, the study wasn't able to capture the effects of genetic diversity on inflammatory responses. In addition, different species have distinct microbiotal compositions, which could contribute to differential immune responses. And, unlike the mice, the human patients were given medical care that might have altered their transcriptional responses. Even considering these factors, the disparity between mouse models of inflammatory disease and their human counterparts raises red flags for these heavily used mouse models in translational research. The differences observed in this study may also go some way to explaining the lack of success in developing therapeutics for inflammatory diseases such as sepsis on the basis of targets identified in mouse models (Nat. Med. 18, 998, 2012), as gene expression patterns would be expected to affect drug sensitivities.

Although the message that mice are an imperfect model for human disease is far from new, these recent results should prompt some soul-searching among disease researchers. As a translational journal, Nature Medicine focuses on understanding and treating human disease, and we put a strong emphasis on human data and the use of in vitro or animal models that are as faithful as possible to the human condition and the most appropriate for the questions being asked.

When selecting a model, some issues that should be considered are whether it reproduces the major clinical symptoms of the human disease, whether the same cells and/or tissues are affected, whether the same genes and molecular pathways are involved and whether the disease proceeds over a similar time course to that in humans. In instances in which mice are not natural hosts of pathogens that infect humans, alternatives such as nonhuman primates may be better suited to studying certain aspects of pathogenesis or to test novel therapeutics.

It's not a simplistic question of whether mice are 'good' or 'bad'; mice undoubtedly have a useful place in translational research. But it is important to realize where such animal models are inherently limited and to bridge the gaps between human patients and the animal model. Initiatives such as the Collaborative Cross (Nat. Gen. 36, 1133-1137, 2004), which has generated a panel of mice to reflect the genetic diversity seen in human populations, might improve the modeling of diseases in mice, particularly complex, multigenic conditions, and allow more nuanced investigation of gene-environment and gene-pathogen interactions. There is also much interest in the development of 'humanized' mice containing human genes, cells, tissues and/or organs, which, for example, have been used to elucidate pathogenic processes occurring in autoimmune conditions including type 1 diabetes and multiple sclerosis (Nat. Med. 18, 66-70, 2012).

In terms of refining models, one of the most logical places to start would be the human situation. In some cases, we need better definitions of clinical phenotypes, especially in heterogeneous or overlapping conditions such as neurodevelopmental and psychiatric diseases. Disease researchers also need to place more emphasis on rigorously defining molecular alterations in human patients that can be quantified, such as genetic, epigenetic, transcriptional and protein changes. This type of information may aid the mapping of the key pathways involved in human disease, and may also allow investigators to systematically analyze how faithfully existing models recapitulate these pathways, as in the recent PNAS study, or even build better ones. Rather than overrelying on animal models to understand what happens in humans, isn't it time to embrace the human 'model' to move forward? 